\title{
Watching two conjugated polymer chains breaking each other when colliding in solution
}

\author{
Yuxi Tian ${ }^{1}$, Marina V Kuzimenkova ${ }^{1}$, Mingyi Xie ${ }^{1}$, Matthias Meyer ${ }^{1}$, Per-Olof Larsson ${ }^{2}$ \\ and Ivan G Scheblykin ${ }^{1}$
}

While collision theory successfully describes the kinetics of chemical reactions, very little is known about the processes at the molecular level, especially if the reacting molecules are large. In this study, using single-molecule spectroscopy, we visually observed that collision between two conjugated polymer (CP) molecules in solution leads to simultaneous rupture of both chains. In addition to opening up the possibility of monitoring chemical processes in solution at the single-molecule level, these results demonstrate that mechanical bending of two stiff conjugated backbones against each other (the effect of leverage) by Brownian motion can weaken the chemical bond and markedly accelerate photochemical oxygen-induced chain scission by at least 20 times. The catalytic effect of the chain bending is also enhanced by a prolonged interaction between the chains owing to their entanglement. These findings are important for the solution processing of CPs in their application in organic electronics, for understanding the degradation mechanisms in CPs and for the development of new catalysts based on mechanical interactions with target molecules.

NPG Asia Materials (2014) 6, e134; doi:10.1038/am.2014.91; published online 3 October 2014

\section{INTRODUCTION}

Understanding the chemical reaction mechanism at the molecular level is of great importance for designing new synthetic routes and developing new materials. After hundreds of years of practical chemistry, the mechanisms of many reactions have been qualitatively understood from the viewpoint of the electric properties of the reactants, such as the nucleophilicity and electrophilicity. ${ }^{1}$ Collision theory was developed to describe the reaction kinetics at the beginning of the past century. ${ }^{2}$ For the reaction to occur, the reactant molecules must collide with each other with a proper orientation and sufficient kinetic energy, as described by Evans and Polanyi ${ }^{3}$ in their transition state theory. Although these approaches are based on mechanical interactions, their applications are often possible only phenomenologically simply because there is no experimental data revealing the mechanistic picture of the interactions between molecules of complicated geometries. Does the collision here only provide the chance of contact between the reactants or is a collision-induced mechanical force also involved? Is there any marked change of the molecular conformation that precedes the reaction?

Indeed, more than a hundred years ago, it was discovered that a mechanical force can have a role in chemical reactions; this discovery gave birth to the field of mechanochemistry. ${ }^{4,5}$ Excellent mechanochemical studies have been performed on large biomolecules and polymers, even at the single-molecule level. ${ }^{6-8}$ However, mechanochemistry, as it is usually understood, requires the application of an additional force onto the reactants via, for example, pressure and flow gradients induced by ultrasound or special flow cells.
Is it possible that the mechanical force from the reactants themselves (such as those generated by thermal diffusion) can also have important roles? On the one hand, this possibility is evident as the reactions are temperature dependent, and temperature means kinetic energy. On the other hand, we do not understand the details of how the mechanical forces created by collision lead to a particular reaction. The details are especially important when reactions between large molecules are considered because the complicated conformations of large molecules, such as conjugated polymers (CPs), could be largely involved in such interactions, affecting the mechanical forces. Understanding the reaction mechanisms between CP chains is of great importance for their applications in organic electronics because CPs are widely used commercially as materials for displays, batteries, organic solar cells, organic light-emitting diodes and even biosensors because of their unique electrical and optical properties. ${ }^{9-11}$

Here, we report real-time visual observations by fluorescence microscopy of collisions between two individual CP molecules in solution leading to the simultaneous rupture of both polymer chains. Our most striking observation is that the chains break apart when they collide with each other simply because of their Brownian motion. Unlike the typical conditions for mechanochemistry, we did not use any externally induced shear forces, as discussed in section 4 in the Supplementary Information. Based on the statistical analysis of many collision events, we propose that mechanical force does have a crucial role in the chain rupture of both polymer molecules and that the force is generated by the crossing of two stiff, long chains undergoing random thermal motion. The entanglement of the chains and the 

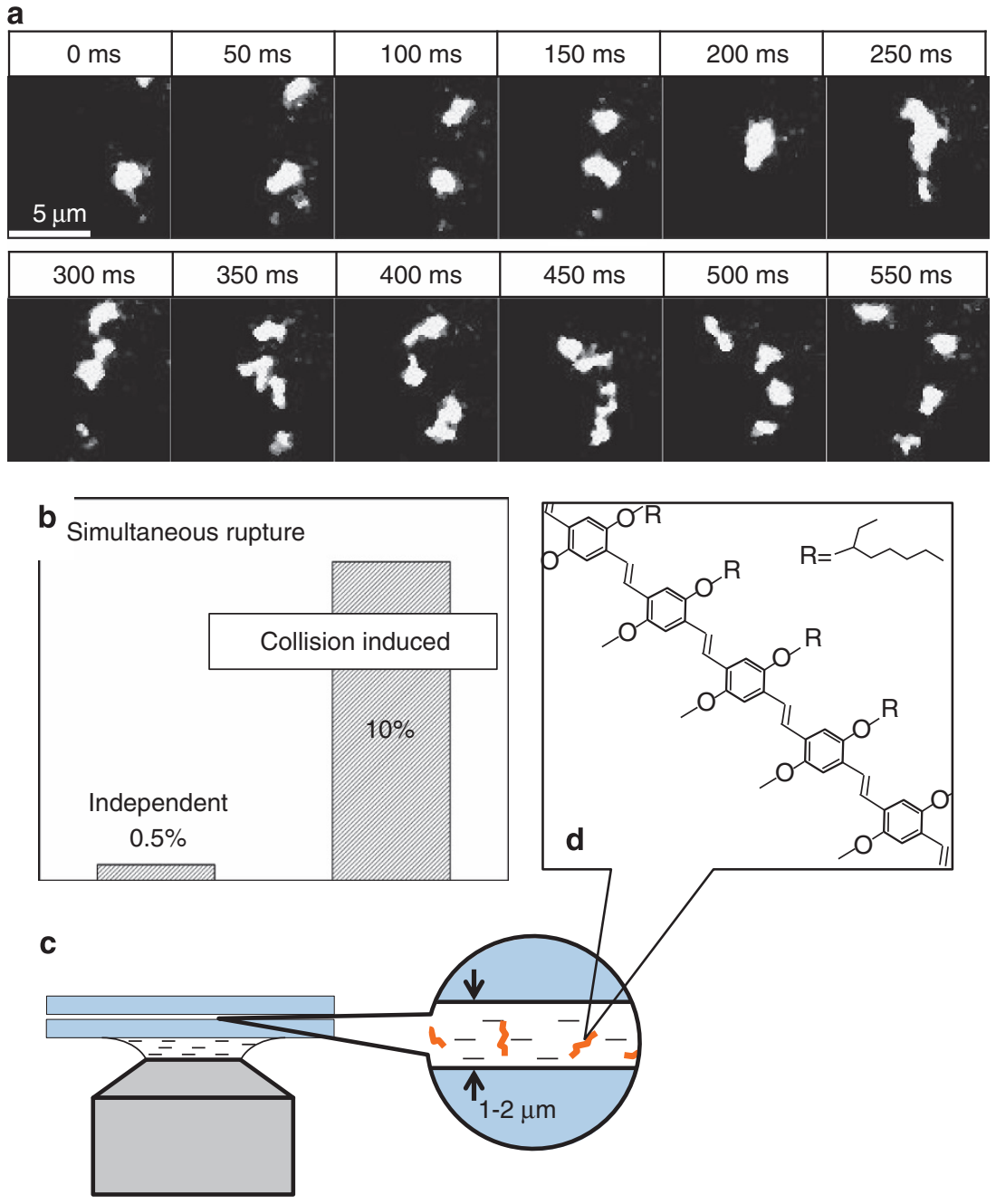

Figure 1 Diffusion and collision of poly(2-methoxy-5-(2-ethylhexyloxy)-1,4-phenylenevinylene) (MEH-PPV). (a) Sequential snapshots following two diffusing MEH-PPV chains, which collide with each other and split into four fragments. The image contrast was enhanced; see Supplementary Figure S1 for the raw data. (b) The probability of independent and collision-induced simultaneous rupture of two chains over the total visual collision events. (c) Experimental setup: a toluene solution containing MEH-PPV and 1\% polymethylmethacrylate (PMMA) was trapped in a 1- to 2- $\mu$ m-thick layer between two glass coverslips. (d) Chemical structure of MEH-PPV.

leverage effect that is possible because of the stiffness of the $\pi$-conjugated backbone can generate a bending stress at the contact point of the chains. The bending stress catalyzes the subsequent photo-oxidation inducing chain rupture.

Our visual observations were performed using single-molecule spectroscopy (SMS), which enables the observation of fluorescence from individual molecules ${ }^{12,13}$ and thereby the identification of the location of molecules in space and the monitoring of their dynamics and fluorescence properties over time. Real-time tracking at the singlemolecule level has been used to reveal biologic processes. ${ }^{14-16}$ However, in spite of its great success in biologic studies, SMS usage in chemistry remains rather scarce. ${ }^{17}$ The chief reason for this scarcity may be a difference in traditions: while biologists are used to working with individual objects that can be observed under a microscope, in chemistry, one traditionally thinks in terms of concentrations.

SMS provides the opportunity to observe directly chemical reactions that involve the changing fluorescence characteristics of chromophores. Fluorescence blinking and bleaching of an individual chromophore are examples of photo-induced electron-transfer reactions. ${ }^{18-21}$ To observe the dynamics of the same single molecule on the scale of seconds, the molecule must be immobilized on a surface or embedded into a transparent polymer matrix. The chemical reactions of single molecules have also been directly observed using scanning probe microscopy, ${ }^{22}$ a technique that is limited to the investigation of surfaces and molecules attached to surfaces. The need for surface immobilization is obviously a great limitation of these methods, as the majority of chemical reactions occur in the liquid phase (solution or melt).

To better understand the mechanism of the chemical reactions at the molecular level in solution, the ability to monitor single molecules in solution would be of great benefit. Fluorescent single molecules can indeed be studied in liquid solutions using fluorescence correlation spectroscopy (FCS); however, FCS cannot be used to follow the same molecule over time and can only provide statistics because every time a different molecule crosses through the focal spot, a signal is produced. ${ }^{23,24}$ 
Here, we aimed to visualize and follow the same single polymer molecules in solution. Recently, the observation and spectroscopy of individual CP chains in semiliquid environments have been demonstrated using solvent vapor annealing or viscous solutions. ${ }^{25,26}$ In this work, we went one step further and visually monitored individual polymer chains in liquid toluene. Unlike all previous studies, ${ }^{27}$ we concentrate not on the chain's photophysics but on the reaction between individual CP chains, which opens a new avenue for applying SMS in chemistry because the reacting molecules can be directly observed in solution owing to their bright fluorescence. We used widefield fluorescence microscopy for real-time observation of individual poly(2-methoxy-5-(2-ethylhexyloxy)-1,4-phenylenevinylene) (MEHPPV) polymer chains with a high molecular weight (ca. $800 \mathrm{kDa}$, $2 \mu \mathrm{m}$ long when stretched) in a very thin $(1-2 \mu \mathrm{m})$ layer of solution (Figure 1c).

\section{MATERIALS AND METHODS}

\section{Sample preparation and experimental setup}

A stock solution of MEH-PPV $\left(0.1 \mathrm{mg} \mathrm{ml}^{-1}\right)$ was prepared by dissolving a suitable amount of MEH-PPV $\left(M_{\mathrm{n}}=150-250 \mathrm{kDa}\right.$; Sigma-Aldrich, St Louis, MO, USA) in tetrahydrofuran. Gel-permeation chromatography was performed with a Waters Styragel HR 5 column $(7.8 \times 300 \mathrm{~mm})$ run at $0.5 \mathrm{ml} \mathrm{min}{ }^{-1}$ (tetrahydrofuran). MEH-PPV was collected as a fraction between 12 and 14 min, corresponding to a molecular weight of $2 \mathrm{MDa}$ relative to polystyrene (PS) standards. The polydispersity index was 1.11 . To assess the absolute molecular weight of the MEH-PPV used, the fact that MEH-PPV is much stiffer than PS needs to be considered. According to the literature, ${ }^{28}$ the molecular weight relative to PS must be divided by a factor between 2 and 3, yielding an estimated absolute molecular weight of $800 \mathrm{kDa}$. Such polymer chains are $\sim 2 \mu \mathrm{m}$ in length when stretched and contain $\sim 3100$ monomer units.

This fraction was then diluted to a concentration of $\sim 10^{-10} \mathrm{M}$ using toluene (anhydrous, 99.8\%). This solution was then further diluted by an additional factor of 10 using the matrix solution ( $1 \%$ by weight of polymethylmethacrylate (PMMA) in toluene, $M_{\mathrm{n}}=996 \mathrm{kDa}$ (Sigma-Aldrich), purified additionally by precipitation). PMMA was used to increase the viscosity of the solution and slow the diffusion of MEH-PPV, which enabled us to follow individual MEHPPV molecules in solution. PMMA also prevents the surface attachment of MEH-PPV on glass surfaces. The final mixture solution was placed between two coverslips, forming a cell with a sandwich structure (see Figure 1c). The thickness of the final solution layer was $1-2 \mu \mathrm{m}$ with a concentration of $\sim 10^{-11} \mathrm{M}$, corresponding to an optical density $\mathrm{OD}_{514 \mathrm{~nm}} \approx 6 \times 10^{-4}$ in a $1 \mathrm{~cm}$ cell. The samples were prepared in air, unless otherwise indicated.

\section{Single-molecule movies}

The samples were studied at ambient conditions using a home-built wide-field fluorescence microscope. The 458-nm laser line from an Ar-ion CW laser was used for excitation. The excitation laser power intensity was $10 \mathrm{~W} \mathrm{~cm}^{-2}$, unless stated otherwise. The fluorescence was collected using an oil-immersion objective lens (Olympus UPlanFLN $\times 60$, N.A. 1.25 ) and imaged on a charge-coupled device camera (ProEM:512B; Princeton Instrument, Trenton, NJ, USA). The movies were taken with an exposure time of $50 \mathrm{~ms}$ per frame.

\section{RESULTS}

We monitored the diffusion, collision and scission of MEH-PPV polymer chains on the single-molecule level. Figure 1a shows the diffusion and collision of two MEH-PPV polymer chains in a thin solution layer. In this example, the collision led to the splitting of both chains into two parts, that is, four fluorescent objects were generated from two (see also Supplementary Movie 1). The total fluorescence intensity of the polymer pieces after the collision remains approximately the same as the total intensity of the two polymer chains before the collision, which indicates that the four pieces are fragments of the two parent CP chains (see Supplementary Figure S2). The fact that we were able to see all the pieces of the chains after the rupture means that the splitting occurred close to the center of the chains, similar to the rupture of polymers by ultrasound or flow gradient. ${ }^{4}$ As will be discussed below, the probability of simultaneous rupture of two chains is at least 20 times larger if they collide than if no interaction occurs.

The experimental setup had some limitations. Owing to the spatial resolution determined by light diffraction and the fast motion of the molecules, we could not be sure that the molecules indeed physically collided, even though the molecules appeared at the same position in the image. To avoid any confusion, we hereafter use the term 'visual collision' to denote the overlapping of two molecules in the image and

\begin{tabular}{|c|c|c|c|c|c|c|}
\hline $0 \mathrm{~ms}$ & $50 \mathrm{~ms}$ & $100 \mathrm{~ms}$ & $150 \mathrm{~ms}$ & $200 \mathrm{~ms}$ & $250 \mathrm{~ms}$ & $300 \mathrm{~ms}$ \\
\hline \hline $5 \mu \mathrm{m}$ & & & & & & \\
\hline & 0 & 0 & & & 0 \\
\hline
\end{tabular}

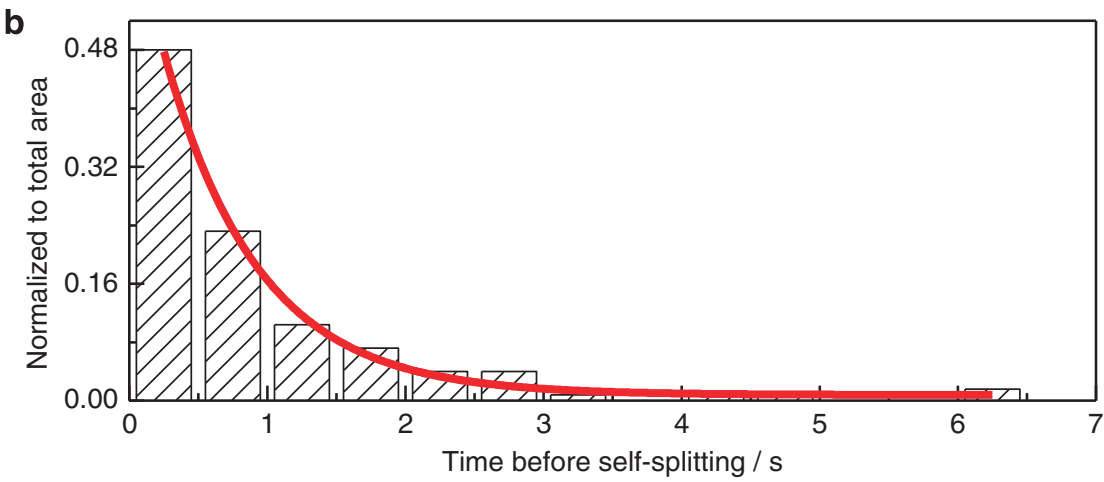

Figure 2 Splitting of a single polymer chain into fragments. (a) Snapshots illustrating self-splitting of a single poly(2-methoxy-5-(2-ethylhexyloxy)-1,4phenylenevinylene) (MEH-PPV) chain. The contrast was enhanced; see Supplementary Figure S3 for the raw data. (b) Histogram of the survival time of individual polymer chains (the molecules that participated in visual collision were excluded for this analysis). The solid line represents a monoexponential fit of the histogram. 
the term 'physical collision' for those molecules that actually physically interacted with each other. Using a simple Monte Carlo simulation, we confirmed that even with the smallest value $(12 \mathrm{~nm})$ for the reported Kuhn lengths of MEH-PPV, ${ }^{29}$ we have a high probability of physical collisions (see section 12 in Supplementary Information). Note that the chains were not large enough for their shape to be resolved in the image. However, owing to the movement of the molecules during the exposure time $(50 \mathrm{~ms})$, the chains appear as randomly shaped spots.

We also observed some collisions resulting in three fluorescent fragments. The collisions that generated four objects constitute 'simultaneous ruptures', and the collisions that generated three objects were considered to be 'one-chain ruptures', which means that only one of the chains split. We analyzed almost 200 visual-collision events between two chains, of which $13 \%$ involved one-chain rupture (see section 6 in Supplementary Information for discussion), 10\% involved the simultaneous rupture of both chains and $77 \%$ showed no effect at all. These numbers will be discussed below. Note that we did not observe any aggregation induced by collisions.

Chain rupture not only occurred when two chains visually collided. The MEH-PPV polymers also split independently without visual collision, as observed in Figure 2a (see also Supplementary Movie 2). Such self-splitting events occurred for almost all the polymer chains, including subchains (pieces from the chain rupture). The splitting continued until either the molecule diffused out of the excitation area or all the pieces were too small to be observed.

We measured the survival time for each molecule exposed to the excitation light by counting the number of frames from the molecule entering the excitation area until the splitting occurred. The survival time histogram for almost 200 molecules is shown in Figure 2b. A monoexponential fit yields a lifetime of the polymer chain before the self-splitting of $0.7 \mathrm{~s}$ under the particular conditions of the experiment. This number will be needed below for statistical analysis of the reactions between individual chains. Using this number and the excitation power density, we can roughly estimate a quantum yield of MEH-PPV photo-induced chain scission of $3 \times 10^{-7}$ in air-saturated toluene. This result means that for MEH-PPV molecules with $M_{\mathrm{n}}$ of $800 \mathrm{kDa}$, the molecular weight can decrease by $50 \%$ within $2 \mathrm{~h}$ at the room light conditions (see section 7 in Supplementary Information for details).

As MEH-PPV solution exhibits good stability in the dark, enabling storage of the long polymer chains in solution for days, we propose that the splitting in the absence of collisions must be a photo-induced chemical reaction. Faster splitting under elevated excitation power also supports this hypothesis.

The CP photodegradation mechanism discussed in the literature involves oxygen: a triplet excitation of the polymer is quenched by an oxygen molecule, which enters a highly reactive singlet state. ${ }^{30-32}$ Then, this singlet oxygen molecule attacks the chemical bonds of the polymer. The long lifetime of the triplet state of MEH-PPV $\left(\sim 100 \mu\right.$ s in deoxygenated solution $\left.{ }^{33}\right)$ provides enough time for such a reaction to occur. To verify the role of oxygen in the MEH-PPV chain rupture, we also performed an experiment under oxygen-free conditions (see section 8 Supplementary Information for details). As observed in Supplementary Movie 3, the self-splitting of the MEHPPV chains is significantly suppressed. Most of the molecules did not split at all while remaining in the excitation area. Therefore, the chain rupture is indeed a photochemical reaction involving oxygen.

\section{DISCUSSION}

We visually observed that collision events resulted in the simultaneous rupture of both chains, generating four fragments. Could this simultaneous rupture be because of two independent one-chain rupture events that by coincidence occurred within one frame? The answer is no. Considering that the lifetime of an independent polymer chain is $0.7 \mathrm{~s}$, which corresponds to a $7 \%$ chance of one-chain rupture per frame $(50 \mathrm{~ms})$, the chance of two molecules splitting independently within one frame is only $\sim 0.5 \%$ (see section 5 in Supplementary Information for calculation). This value is 20 times less than the rate of simultaneous rupture observed experimentally $(10 \pm 2 \%$; Figure $1 b)$. Note here that this $20 \times$ enhancement is an underestimation because (i) not all visual collisions necessarily constitute physical collisions and (ii) the collision time may be significantly shorter than our integration time of $50 \mathrm{~ms}$. Therefore, we conclude that simultaneous chain rupture is caused by physical collision. This conclusion leads to another conclusion: the physical collision catalyzes the photochemical reaction of chain rupture under the given experimental conditions. To the best of our knowledge, this observation represents the first time that a collision process that catalyzes chemical reactions was visually observed at the individual molecule level.

One may have an initial suspicion that the splitting of the fluorescence spots could be the result of the dissociation of noncovalently bound MEH-PPV aggregates. It is known that such aggregates exist in solutions, ${ }^{24,34}$ and we have also studied the chain aggregation of MEH-PPV in detail, which will be published elsewhere. In this study, to avoid aggregation, we collected a fraction from a gelpermeation chromatography run, in which the injected MEH-PPV sample exhibited a concentration that was orders of magnitude lower than that normally used for gel-permeation chromatography fractionation. The fraction was then further diluted to the single-molecule level to reach $\sim 10^{-11} \mathrm{M}$, which corresponds to an optical density $\mathrm{OD}_{514 \mathrm{~nm}} \sim 6 \times 10^{-4}$ in a $1 \mathrm{~cm}$ cell. Under such conditions, we are confident that the bright spots we observed in the solution are single polymer chains. In addition, further evidences convinced us that the splitting is chain rupture rather than disaggregation. First, we would not expect a disaggregation process to be photo-induced because the aggregation does not involve any excited electronic states. Second, the collision of polymer chains should rather promote aggregation than dissociation, which was never observed in any of the 200 studied collisions cases. Finally, we observed that the splitting rate of $\mathrm{MEH}-$ PPV depends on the concentration of oxygen, which should not occur if the process is disaggregation.

It is surprising that a collision that results solely from Brownian motion can affect the chain rupture. As far as the authors are aware, such an effect has not been reported before. Indeed, the average energy available from thermal motion is only $k_{\mathrm{B}} T / 2\left(c a .10^{-21} \mathrm{~J}\right.$ at $\left.300 \mathrm{~K}\right)$ per degree of freedom. Clearly, this energy is too small to break the bond between carbon atoms of CPs with binding energies of $\sim 8 \times 10^{-19} \mathrm{~J}^{35}$ However, a direct comparison of the energies is not fully correct because if one follows the thermal fluctuations of a single polymer chain over a very long time, there is a finite chance of observing a fluctuation with much larger energy in a particular degree of freedom than $k_{\mathrm{B}} T$. This idea is used for calculations of chain rupture because of bond stretching in the modeling of thermally induced polymer degradation. ${ }^{36}$ Below, we will see that it is not only the amount of energy but also the way in which the energy is applied to the system that is of great importance.

CPs are special polymers that are much stiffer than ordinary flexible organic polymers, for example, polyethylene, PMMA and PS. This 
stiffness arises because the connection between carbon atoms in CPs is not a single $\sigma$ bond, as in flexible organic polymers, but the combination of $\sigma$ and $\pi$ bonding that originates from the delocalization of $\pi$ electrons along the length of the polymer, which prevents the rotation of the chain along the chemical bonds. The flexibility of a polymer can be described by the length of its Kuhn segment; these segments are considered to be freely joined with each other within a whole polymer molecule. The Kuhn length of MEH-PPV was reported to be $12-65 \mathrm{~nm}$, which is more than an order of magnitude larger than that for ordinary flexible polymers $(1-2 \mathrm{~nm})$, indicating that $\mathrm{MEH}-$ PPV resists coiling owing to its stiffness. ${ }^{29} \mathrm{We}$ shall explore below how their stiffness can be important for the collision process between the CPs by Brownian motion.

The ancient scientist Archimedes said: 'Give me a lever and a place to stand on, I will move the earth'. Thus, the problem of not having enough force to break the bond by pulling two atoms apart can be solved using the principle of leverage. Everyday experience (e.g., breaking tree branches) tells us that it is much easier to break a stiff object by bending it than by stretching. Bending has also been suggested as the mechanism of breaking of stiff carbon nanotubes by ultrasound-induced pressure gradient. ${ }^{37}$ The bending should be performed against as small an object as possible (e.g., a knee in the case of tree branches) and, to minimize the force, with as much leverage as possible. For colliding MEH-PPV chains, the 'knee' is the contact point between the two chains, and the lever is the long stiff chain itself. The chain interaction time $(\mathrm{ms})$ is many orders of magnitude larger than the characteristic time of thermally induced molecular vibrations (ps). Therefore, statistically, Brownian fluctuations could sometimes create a large torque at the contact point (Figure 3). Bending a polymer chain enough to break it remains unlikely; however, as mentioned above, many other factors are involved in the chain rupture, including photoexcitation and oxygen. Even a small bending stress on the stiff polymer chain would perturb the $\pi$ conjugation and increase the total energy, as illustrated by Walsh's rules for small molecules, ${ }^{38}$ and make the polymer more susceptible to photo-oxidation and subsequent scission of the chains. Moreover, the bending of the conjugated backbone could also increase the intersystem crossing yield. A higher generation yield of triplet excitations proportionally increases the chance of the reaction involving singlet oxygen. Note that this breaking mechanism is an exclusive property of CPs or of polymers with stiff backbones in general, because flexible polymers such as PMMA, PS and polyethylene do not have any appreciable resistance for bending, and therefore, bending cannot disturb their chemical bonds.

How can we explain the simultaneous rupture of both chains? One possible explanation is that when the chains are bent, the stress remains at the second chain for some time even after the first chain is already broken. The residual bending stress relaxes with the chainrelaxation time $(\sim 1 \mathrm{~ms})$, which is long enough to ensure the chain rupture (see section 11 in Supplementary Information for the estimation). An alternative possibility is that when the first chain breaks, the radicals formed after the bond scission ${ }^{34}$ immediately attack the second chain, thus inducing its splitting.

We must admit that we cannot directly prove the bending hypothesis discussed above; however, as described below, there is no probable alternative explanation.

We begin our logic from the experimental fact that physical interaction between chains markedly accelerates the chain rupture. Because the only difference between two free chains and two colliding chains is the presence of a contact point, the splitting reaction must occur at the contact point. The lifetime of the 'contact state' must be on the order of the chain relaxation time, which is in the millisecond time range for long polymers (see section 11 in Supplementary Information for the estimation).

Having a contact point presumes mechanical force; otherwise, there would be no contact. Therefore, the question is not whether mechanical interaction is present or not but instead concerns the interaction type (bending or stretching) and whether this interaction is crucial or not for the chain rupture.

Let us assume that the mechanical stress is not important and that the reaction solely occurs because two conjugated segments are brought into close contact. This mechanism appears to be highly unlikely because MEH-PPV can exist as a pristine film or as chain aggregates, which are chemically stable materials. In such materials, every monomer unit of the conjugated backbone is located near other monomer units. Therefore, the chances for chains breaking under such conditions would be thousands of times higher than for two chains only having one contact point. In brief, if this mechanism occurred, MEH-PPV would rapidly break apart into monomers when maintained in a solid state.

The mechanical force can lead to stretching or bending. Stretching forces are always present even in individual chains simply because of

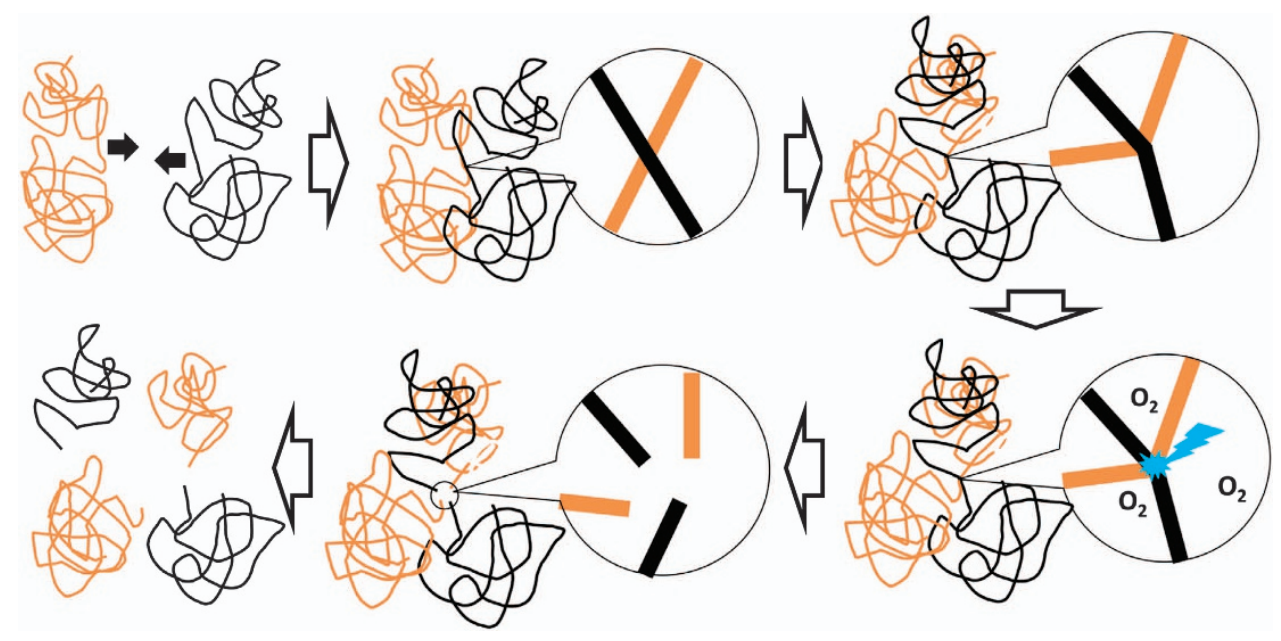

Figure 3 Schematic depiction of the collision process of two poly(2-methoxy-5-(2-ethylhexyloxy)-1,4-phenylenevinylene) (MEH-PPV) chains. Collisions induce the simultaneous rupture of both chains because of a photochemical reaction catalyzed by the high tension at the contact point induced by a leverage effect. 
thermal fluctuations. There is no reason to expect a marked increase of the stretching force when two molecules become entangled. Therefore, we exclude stretching as a major contributor. Unlike stretching, bending stress is much more probable for colliding chains than for solitary chains. While bending stress is inherent to colliding entangled chains, solitary chains cannot experience the same stress simply because crossing of their chain segments is unlikely because of the self-avoidance principle. ${ }^{39}$ Although the probability is lower, we cannot exclude the possibility of self-splitting due to the similar mechanical interaction between different segments of the same chain.

In summary, having eliminated all viable alternative interpretations, we conclude that collision-induced bending is the most probable explanation of the observed effects.

\section{CONCLUSIONS}

The types of chemical reactions that can be investigated at the singlemolecule level are still in the discovery phase. ${ }^{17}$ Most chemical reactions studied by SMS so far were reactions on glass surfaces or on catalysts. ${ }^{40,41}$ We demonstrate that SMS can be used to study reactions in the solution phase, which opens up a new dimension of chemical processes for investigation. The real-time imaging of single molecules in free solution can be used for monitoring in situ chemical reactions involving macromolecules, biomolecules and nanoparticles.

The goal of single-molecule chemistry is to provide a clear mechanistic view of chemical processes by eliminating ensemble averaging, which is essential for their understanding. Our observation is an educative illustration on the single-molecule level of the classical activated-complex theory of chemical reactions proposed by Eyring and Polanyi in 1935. ${ }^{3,42}$ Two molecules collide and form bent photoexcited states (an activated complex) that react further with oxygen. The suggested mechanism based on molecular stiffness and the leverage effect is important for the applications of these materials in organic electronics and for understanding their degradation mechanisms and may also open up new avenues for solution catalysis, for example, the use of specially designed 'molecular knives' (e.g., small pieces of graphene).

\section{CONFLICT OF INTEREST}

The authors declare no conflict of interest.

\section{ACKNOWLEDGEMENTS}

This study was financially supported by The Swedish Research Council, the Knut \& Alice Wallenberg Foundation, the Crafoord Foundation and the Carl Trygger Foundation.

1 Wade, L. Organic Chemistry (Pearson Prentice Hall, Upper Saddle River, NJ, 2006).

2 Bawn, C. E. H. William Cudmore McCullagh Lewis. 1885-1956. Biogr. Mem. Fellows R. Soc. 4, 192-203 (1958).

3 Evans, M. G. \& Polanyi, M. Some applications of the transition state method to the calculation of reaction velocities, especially in solution. Trans. Faraday Soc. 31 875 (1935).

4 Beyer, M. K. \& Clausen-Schaumann, H. Mechanochemistry: the mechanical activation of covalent bonds. Chem. Rev. 105, 2921-2948 (2005).

5 Caruso, M. M., Davis, D. A., Shen, Q., Odom, S. A., Sottos, N. R., White, S. R. \& Moore, J. S. Mechanically-induced chemical changes in polymeric materials. Chem. Rev. 109, 5755-5798 (2009).

6 Cecconi, C., Shank, E. A., Bustamante, C. \& Marqusee, S. Direct observation of the three-state folding of a single protein molecule. Science 309 , 2057-2060 (2005)

7 Hickenboth, C. R., Moore, J. S., White, S. R., Sottos, N. R., Baudry, J. \& Wilson, S. R. Biasing reaction pathways with mechanical force. Nature 446, 423-427 (2007).

8 Piermattei, A., Karthikeyan, S. \& Sijbesma, R. P. Activating catalysts with mechanical force. Nat. Chem. 1, 133-137 (2009).
9 Friend, R. H., Gymer, R. W., Holmes, A. B., Burroughes, J. H., Marks, R. N., Taliani, C. Bradley, D. D. C., Santos, D. A., Dos, Bredas, J. L, Logdlund, M. \& Salaneck, W. R. Electroluminescence in conjugated polymers. Nature 397, 121-128 (1999).

10 Grimsdale, A. C., Chan, K. L., Martin, R. E., Jokisz, P. G. \& Holmes, A. B. Synthesis of light-emitting conjugated polymers for applications in electroluminescent devices. Chem. Rev. 109, 897-1091 (2009).

11 Samuel, I. D. W. \& Turnbull, G. A. Organic semiconductor lasers. Chem. Rev. 107, 1272-1295 (2007).

12 Moerner, W. E. \& Orrit, M. Illuminating single molecules in condensed matter. Science 283, 1670-1676 (1999).

13 Vanden Bout, D. A., Yip, W.-T., Hu, D., Fu, D., Swager, T. M. \& Barbara, P. F. Discrete intensity jumps and intramolecular electronic energy transfer in the spectroscopy of single conjugated polymer molecules. Science 277, 1074-1077 (1997).

14 Gebhardt, J. C. M., Suter, D. M., Roy, R., Zhao, Z. W., Chapman, A. R., Basu, S., Maniatis, T. \& Xie, X. S. Single-molecule imaging of transcription factor binding to DNA in live mammalian cells. Nat. Methods 10, 421-426 (2013).

15 Guan, Y., Wang, Z., Cao, A., Lai, L. \& Zhao, X. S. Subunit exchange of MjHsp16.5 studied by single-molecule imaging and fluorescence resonance energy transfer. J. Am. Chem. Soc. 128, 7203-7208 (2006).

16 Goldsmith, R. H. \& Moerner, W. E. Watching conformational- and photo-dynamics of single fluorescent proteins in solution. Nat. Chem. 2, 179-186 (2010),

17 Cordes, T. \& Blum, S. A. Opportunities and challenges in single-molecule and singleparticle fluorescence microscopy for mechanistic studies of chemical reactions. Nat. Chem. 5, 993-999 (2013).

18 Lin, H., Tabaei, S. R., Thomsson, D., Mirzov, O., Larsson, P. O. \& Scheblykin, I. G. Fluorescence blinking, exciton dynamics, and energy transfer domains in single conjugated polymer chains. J. Am. Chem. Soc. 130, 7042-7051 (2008).

19 Vogelsang, J., Kasper, R., Steinhauer, C., Person, B., Heilemann, M., Sauer, M. \& Tinnefeld, P. A reducing and oxidizing system minimizes photobleaching and blinking of fluorescent dyes. Angew. Chem. Int. Ed. Engl. 47, 5465-5469 (2008).

20 Park, S.-J., Gesquiere, A. J., Yu, J. \& Barbara, P. F. Charge injection and photooxidation of single conjugated polymer molecules. J. Am. Chem. Soc. 126 4116-4117 (2004).

21 Hania, P. R. \& Scheblykin, I. G. Electric field induced quenching of the fluorescence of a conjugated polymer probed at the single molecule level. Chem. Phys. Lett. 414 127-131 (2005).

22 De Oteyza, D. G. Gorman, P. Chen, Y C., Wickenburg, S., Riss, A Mowbray, D. J., Etkin, G., Pedramrazi, Z., Tsai, H. Z., Rubio, A., Crommie, M. F. \& Fischer, F. R. Direct imaging of covalent bond structure in single-molecule chemical reactions. Science $\mathbf{3 4 0}$, 1434-1437 (2013).

23 Widengren, J. \& Rigler, R. Fluorescence correlation spectroscopy as a tool to investigate chemical reactions in solutions and on cell surfaces. Cell. Mol. Biol. (Noisy-le-grand) 44, 857-879 (1998).

24 Wang, D., Yuan, Y., Mardiyati, Y., Bubeck, C. \& Koynov, K. From single chains to aggregates, how conjugated polymers behave in dilute solutions. Macromolecules 46, 6217-6224 (2013).

25 Vogelsang, J., Brazard, J., Adachi, T., Bolinger, J. C. \& Barbara, P. F. Watching the annealing process one polymer chain at a time. Angew. Chem. Int. Ed. Engl. 50, 2257-2261 (2011)

26 Onda, S., Kobayashi, H., Hatano, T., Furumaki, S., Habuchi, S. \& Vacha, M. Complete suppression of blinking and reduced photobleaching in single MEH-PPV chains in solution. J. Phys. Chem. Lett. 2, 2827-2831 (2011)

27 Lupton, J. M. Single-molecule spectroscopy for plastic electronics: materials analysis from the bottom-up. Adv. Mater. 22, 1689-1721 (2010).

28 Becker, K., Gaefke, G., Rolffs, J., Höger, S. \& Lupton, J. M. Quantitative mass determination of conjugated polymers for single molecule conformation analysis: enhancing rigidity with macrocycles. Chem. Commun. (Camb) 46, 4686-4688 (2010).

29 DuBay, K. H., Hall, M. L., Hughes, T. F., Wu, C., Reichman, D. R. \& Friesner, R. A Accurate force field development for modeling conjugated polymers. J. Chem. Theory Comput. 8, 4556-4569 (2012).

30 Radousky, H. B., Madden, A. D., Pakbaz, K. \& Hagler, T. W. Accelerated degradation studies of MEH-PPV. Int. SAMPE Technol. Conf. 1143-1146 (1995).

31 Dam, N., Scurlock, R. D., Wang, B., Ma, L., Sundahl, M. \& Ogilby, P. R. Singlet oxygen as a reactive intermediate in the photodegradation of phenylenevinylene oligomers. Chem. Mater. 11, 1302-1305 (1999).

32 Burrows, H. D., Narwark, O., Peetz, R., Thorn-Csányi, E., Monkman, A. P., Hamblett, I. \& Navaratnam, S. Mechanistic studies on the photodegradation of 2,5-dialkyloxylsubstituted para-phenylenevinylene oligomers by singlet oxygen. Photochem. Photobiol. Sci. 9, 942-948 (2010)

33 Monkman, A. P., Burrows, H. D., Miguel, M. d. G., Hamblett, I. \& Navaratnam, S. Triplet state spectroscopy of conjugated polymers studied by pulse radiolysis. Synth. Met. 116, 75-79 (2001)

34 Nguyen, T.-Q., Doan, V. \& Schwartz, B. J. Conjugated polymer aggregates in solution control of interchain interactions. J. Chem. Phys. 110, 4068 (1999).

35 Röhrig, U. F. \& Frank, I. First-principles molecular dynamics study of a polymer under tensile stress. J. Chem. Phys. 115, 8670 (2001)

36 Fugmann, S. \& Sokolov, I. M. Thermally activated breakdown in a simple polymer model. Phys. Rev. E 81, 031804 (2010).

37 Pagani, G., Green, M. J., Poulin, P. \& Pasquali, M. Competing mechanisms and scaling laws for carbon nanotube scission by ultrasonication. Proc. Natl Acad. Sci. USA 109 11599-11604 (2012).

38 Walsh, A. D. The electronic orbitals, shapes, and spectra of polyatomic molecules. Part I. AH2 molecules. J. Chem. Soc. 0, 2260 (1953). 
39 Werner, E., Westerlund, F., Tegenfeldt, J. O. \& Mehlig, B. Monomer distributions and intrachain collisions of a polymer confined to a channel. Macromolecules 46, 6644-6650 (2013).

40 Roeffaers, M. B. J., Sels, B. F., Uji-I, H., De Schryver, F. C., Jacobs, P. A., De Vos, D. E. \& Hofkens, J. Spatially resolved observation of crystal-face-dependent catalysis by single turnover counting. Nature 439, 572-575 (2006).

41 Zhou, X., Andoy, N. M., Liu, G., Choudhary, E., Han, K. S., Shen, H. \& Chen, P. Quantitative super-resolution imaging uncovers reactivity patterns on single nanocatalysts. Nat. Nanotechnol. 7, 237-241 (2012).

42 Eyring, H. The activated complex in chemical reactions. J. Chem. Phys. 3, 107 (1935). (c) (i) (5)

This work is licensed under a Creative Commons Attribution-NonCommercial-ShareAlike 3.0 Unported License. The images or other third party material in this article are included in the article's Creative Commons license, unless indicated otherwise in the credit line; if the material is not included under the Creative Commons license, users will need to obtain permission from the license holder to reproduce the material. To view a copy of this license, visit http://creativecommons.org/licenses/by-nc-sa/3.0/

Supplementary Information accompanies the paper on the NPG Asia Materials website (http://www.nature.com/am) 